\title{
Molecular genotyping of papillary thyroid carcinoma follicular variant according to its histological subtypes (encapsulated vs infiltrative) reveals distinct $B R A F$ and $R A S$ mutation patterns
}

\author{
Michael Rivera ${ }^{1,5}$, Julio Ricarte-Filho ${ }^{2,5}$, Jeff Knauf ${ }^{2,3}$, Ashok Shaha ${ }^{4}$, Michael Tuttle ${ }^{3}$, \\ James A Fagin ${ }^{2,3,6}$ and Ronald A Ghossein ${ }^{1,6}$ \\ ${ }^{1}$ Department of Pathology, Memorial Sloan-Kettering Cancer Center, New York, NY, USA; ${ }^{2}$ Human Oncology \\ and Pathogenesis Program, Memorial Sloan-Kettering Cancer Center, New York, NY, USA; ${ }^{3}$ Department of \\ Medicine, Memorial Sloan-Kettering Cancer Center, New York, NY, USA and ${ }^{4}$ Department of Surgery, \\ Memorial Sloan-Kettering Cancer Center, New York, NY, USA
}

\begin{abstract}
The follicular variant of papillary thyroid carcinoma usually presents as an encapsulated tumor and less commonly as a partially/non-encapsulated infiltrative neoplasm. The encapsulated form rarely metastasizes to lymph node, whereas infiltrative tumor often harbors nodal metastases. The molecular profile of the follicular variant was shown to be close to the follicular adenoma/carcinoma group of tumors with a high RAS and very low BRAF mutation rates. A comprehensive survey of oncogenic mutations in the follicular variant of papillary thyroid carcinoma according to its encapsulated and infiltrative forms has not been performed. Paraffin tissue from 28 patients with encapsulated and 19 with infiltrative follicular variant were subjected to mass spectrometry genotyping encompassing the most significant oncogenes in thyroid carcinomas: 111 mutations in RET, BRAF, NRAS, HRAS, KRAS, PIK3CA, AKT1 and other related genes. There was no difference in age, gender, tumor size and angioinvasion between encapsulated or infiltrative tumors. Infiltrative carcinomas had a much higher frequency of extrathyroid extension, positive margins and nodal metastases than encapsulated tumors $(P<0.05)$. The BRAF 1799T $>A$ mutation was found in 5 of $19(26 \%)$ of the infiltrative tumor and in none of the encapsulated carcinomas $(P=0.007)$. In contrast, RAS mutations were observed in 10 of $28(36 \%)$ of the encapsulated group (5 NRAS_Q61R, 3 HRAS_Q61, 1 HRAS_G13C and 1 KRAS_Q61R) and in only 2 of $19(10 \%)$ of infiltrative tumors $(P=0.09)$. One encapsulated carcinoma showed a PAX8/PPAR $\gamma$ rearrangement, whereas two infiltrative tumors harbored RET/PTC fusions. Encapsulated follicular variant of papillary thyroid carcinomas have a molecular profile very close to follicular adenomas/carcinomas (high rate of RAS and absence of BRAF mutations). Infiltrative follicular variant has an opposite molecular profile closer to classical papillary thyroid carcinoma than to follicular adenoma/carcinoma (BRAF $>$ RAS mutations). The molecular profile of encapsulated and infiltrative follicular variant parallels their biological behavior (ie, metastatic nodal and invasive patterns). Modern Pathology (2010) 23, 1191-1200; doi:10.1038/modpathol.2010.112; published online 4 June 2010
\end{abstract}

Keywords: BRAF; follicular variant; genotyping; molecular; papillary thyroid carcinoma; RAS

Correspondence: Dr RA Ghossein, MD, Department of Pathology, Memorial Sloan-Kettering Cancer Center, 1275 York Avenue, New York, NY 10021, USA.

E-mail:ghosseir@mskcc.org

Part of this work was accepted as an abstract at the United States and Canadian Academy of Pathology Meeting in Washington, DC, March 2010.

${ }^{5}$ These authors contributed equally to this work.

${ }^{6}$ These authors contributed equally to this work.

Received 2 February 2010; revised 16 March 2010; accepted 24 March 2010; published online 4 June 2010
The papillary thyroid carcinoma family of tumor is defined histologically by the presence of clear, irregular, overlapping nuclei with grooves and pseudoinclusions. In addition to the aforementioned nuclear features, classical ('garden variety') papillary thyroid carcinoma is composed of a variable mixture of papillae and follicles. In contrast, the follicular variant of papillary thyroid carcinoma is almost exclusively arranged in follicles lined by cells with characteristic papillary 
carcinoma nuclei. ${ }^{1}$ The follicular variant of papillary carcinoma presents usually as an encapsulated tumor and less commonly as a partially/non-encapsulated infiltrative neoplasm. ${ }^{2}$ Encapsulated follicular variant of papillary carcinoma is a frequent diagnosis in current surgical pathology practice, being the most common variant of papillary carcinoma. The diagnosis of encapsulated follicular variant is difficult at the cytopathological, frozen section and histological level. This is reflected in considerable interobserver variability. ${ }^{3,4}$ Recent studies have shown that the encapsulated form rarely metastasize to lymph node ( $5 \%$ of cases), whereas infiltrative follicular variant often harbors lymph node (LN) metastases (65\% of patients). ${ }^{2}$ The encapsulated follicular variant is therefore closer in its invasive and nodal metastatic pattern to the follicular adenoma/carcinoma group of tumors, whereas infiltrative follicular variant behaves similar to classical papillary thyroid carcinoma. ${ }^{2}$

In addition, the molecular profile of the follicular variant was shown to be close to the follicular adenoma/carcinoma group of tumors with a high prevalence of $R A S$ and a very low BRAF mutation rate. ${ }^{5}$ However, a comprehensive survey of oncogenic mutations in the follicular variant of papillary thyroid carcinoma according to its encapsulated and non-encapsulated forms has not been performed. In conjunction with available histological and followup data, we show that the delineation of the molecular profile of the follicular variant of papillary thyroid carcinoma according to its subtypes helps better classify these tumors into clinically relevant entities.

\section{Materials and methods}

\section{Patient Population and Inclusion Criteria}

The institutional database was searched for all cases with a diagnosis of thyroid carcinomas treated at Memorial Sloan-Kettering Cancer Center between January 1980 and December 2002. Additional cases were supplied from the personal file of one of us (RAG). The slides from the cases included in the study were examined by two head and neck pathologists with special interest in thyroid neoplasia (RAG and MR). The pathologists were blinded to the clinical outcome of all patients studied. The follicular variant of papillary thyroid carcinomas were defined as follicular lesions almost entirely composed of follicles (up to $99 \%$ of the neoplasm) lined by cells having the characteristic nuclear features of papillary carcinoma (ie, clear, irregular and overlapping nuclei with grooves and pseudoinclusions). These nuclear changes were either diffuse or multifocal (encompassing at least $60 \%$ of the tumor even when multifocal). The nuclear changes were well developed (ie, at least enlarged, irregular hypochromatic nuclei with overlapping and occasional grooves). All follicular variant of papillary thyroid carcinomas (encapsulated and infiltrative) had $\leq 1 \%$ papillary formations. Follicular variant of papillary thyroid carcinomas were designated as encapsulated if totally surrounded by a fibrous capsule and as infiltrative if partially or totally unencapsulated with invasive tongues of tumor-infiltrating nonneoplastic thyroid parenchyma (Figures 1 and 2). Only the follicular variant of papillary thyroid carcinomas of $\geq 1 \mathrm{~cm}$ in greatest size were analyzed. Tumors with high mitotic activity ( $\geq 5$ mitosis/10 high power fields, $\times 400$ ) and/or tumor necrosis were excluded as well as those containing $\geq 30 \%$ tall cells. The latter cell type was defined as having a height at least twice its width with an oncocytic cytoplasm. The study was approved by the institutional review board of the Memorial Sloan-Kettering Cancer Center.

\section{Histopathological Analysis}

The largest dimension of the tumor was recorded as dictated during gross examination. The mitotic rate of the tumor was determined by counting 10 contiguous high power fields $(\times 400)$ using an Olympus microscope (U-DO model BX-40; Olympus America, Melville, NY, USA). Using that microscope type, these 10 high power fields corresponded to $2.4 \mathrm{~mm}^{2}$. Mitotic counts were performed in a focused manner, examining areas that appeared to show greater proliferative activity. Tumor necrosis was defined by a 'comedo-like' appearance composed of degenerating cytoplasm and punctuate, karyorrhectic nuclear debris. The presence of fibroblastic stromal reaction, hemorrhage or an identifiable needle track in the necrotic area was attributable to reaction induced by previous fine needle aspiration, and was therefore not regarded as spontaneous tumor necrosis. When present, the numbers of foci of capsular and vascular invasion were noted. Only complete penetration of the capsule by tumor was regarded as capsular invasion, as described by Lang et al. ${ }^{6}$ The presence of vascular invasion was noted only when such foci were present within or beyond the capsule in accordance with criteria outlined by the Armed Forces Institute of Pathology (AFIP) fascicle. ${ }^{1}$ Only when the invasive focus protruded into the lumen of the vessel in a polypoid manner covered by endothelial cells, or when it was attached to the vessel wall or associated with thrombus formation, was it considered true vascular invasion. Areas of vascular invasion that were closely adjacent to one another were counted as separate foci. The foci of capsular and vascular invasion were subdivided into two categories: focal ( $<4$ invasive foci) and extensive $(\geq 4$ foci).

\section{Clinical Parameters}

The patient's electronic medical records were reviewed for the age at diagnosis, type of surgery 

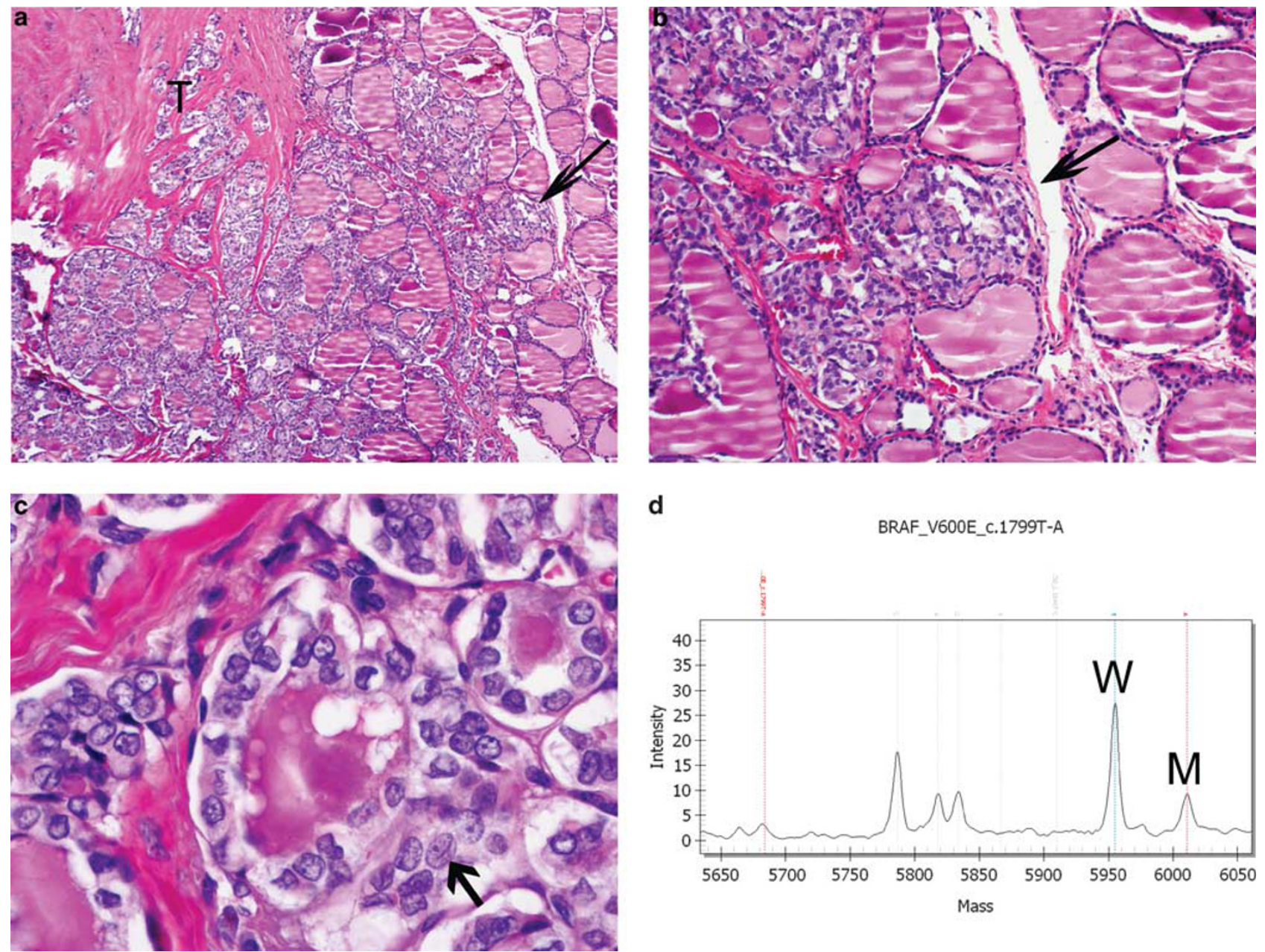

d

BRAF_V600E_C.1799T-A

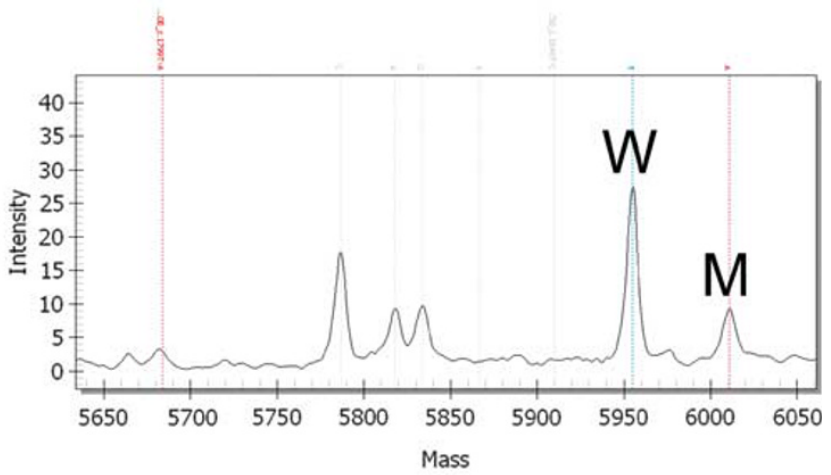

Figure 1 Infiltrative follicular variant of papillary thyroid carcinoma. (a) Low-power view showing tumor (T) growing in a follicular pattern with marked fibrosis and an infiltrating advancing front (arrow). (b) Medium power showing tongues of tumor-infiltrating nonneoplastic thyroid without interposition of a capsule (arrow). (c) High-power view of the nuclear features of papillary thyroid carcinoma: hypochromatic, irregular and overlapping nuclei with grooves (the latter indicated by an arrow). (d) Mass spectrometry traces for BRAF mutation from the tumor. Note the mutant BRAF_T1799A (indicated by M) and wild-type (W) peaks.

and adjuvant treatment, including radioactive iodine therapy. The current disease status was based on a combination of physical examination, biochemical serum markers (thyroid stimulating hormone and thyroglobulin), radioactive iodine dosimetry, cross-sectional imaging and/or positron emission tomography (PET) scanning. The date of initial surgery and last date of follow-up were recorded. The status at last follow-up was recorded as follows: no evidence of disease; alive with disease; dead of other causes; and dead of disease.

\section{Genotyping by Mass Spectrometry}

Four sections of $10 \mu \mathrm{m}$ from each formalin-fixed paraffin-embedded tissue block were subjected to DNA extraction using the PUREGene Genomic DNA purification kit (Gentra, Minneapolis, MN, USA). Mutation detection was performed as described previously. ${ }^{7}$ We used mass spectrometry Sequenom- based genotyping assay (Sequenom Mass array; Sequenom, San Diego CA, USA), which is especially suited for high-throughput genotyping, to interrogate 111 known mutations in 16 different genes: BRAF, RET, NRAS, HRAS, KRAS, PIK3CA, MAP2K1, AKT1, MET, IKBKB, PIK3R5, PRKCZ, RHEB, RPS6KA3, RPS6KB1 and FRAP1. Of note, BRAFK60оE was also included in the detection platform. As the mass spectrometry genotyping assays for codons 12 and 13 of HRAS were not informative, we designed primers for this region and sequenced all the tumors that were wild type for $B R A F, R A S$ mutations or for $R E T / P T C$ rearrangements. ${ }^{7}$

\section{Screening for RET/PTC and PAX8/PPAR $\gamma$ Rearrangements}

We used tumor cDNA as template for quantitative polymerase chain reaction (PCR) to analyze for unbalanced expression of exons 10 and 11 relative 

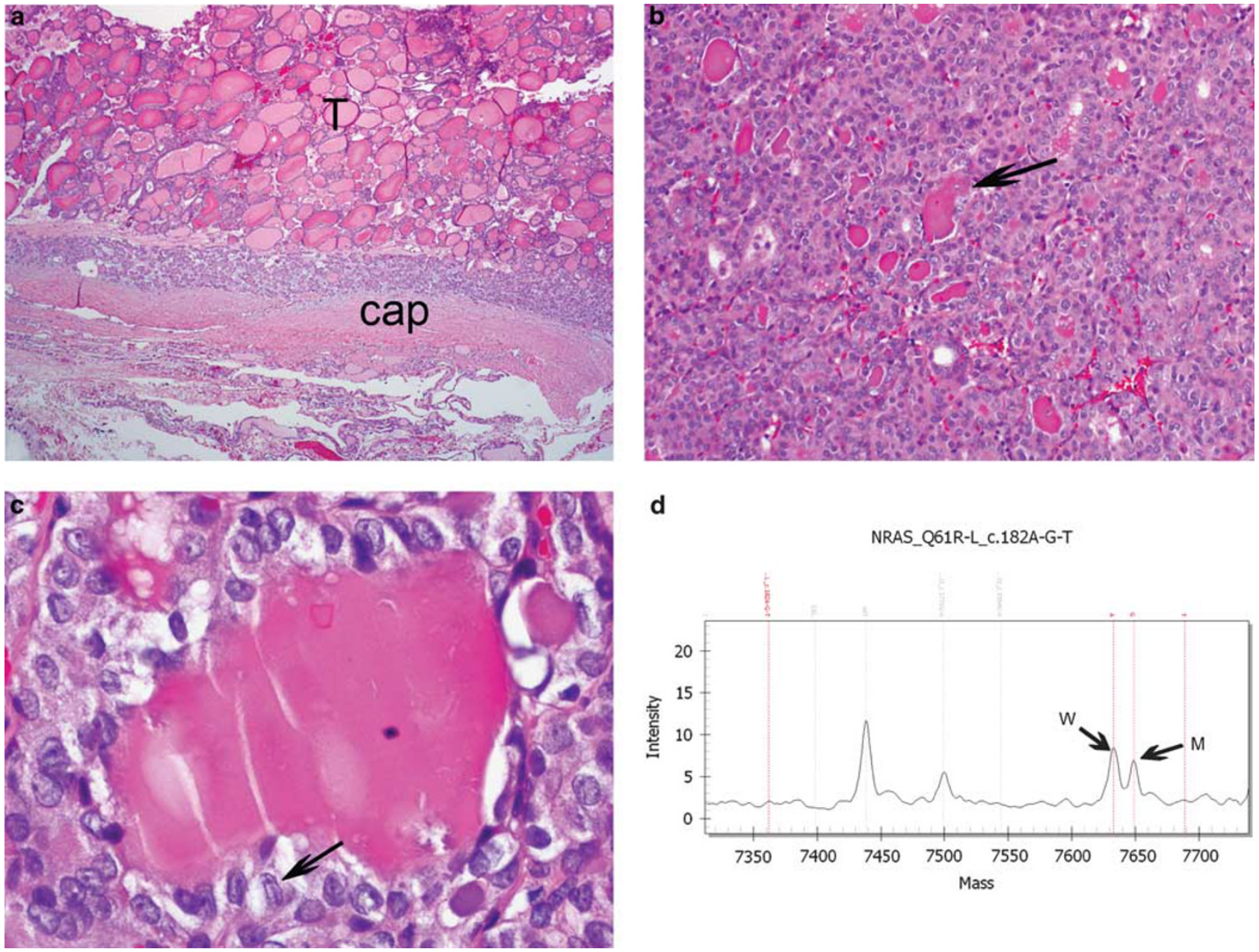

d

NRAS_Q61R-L_C.182A-G-T

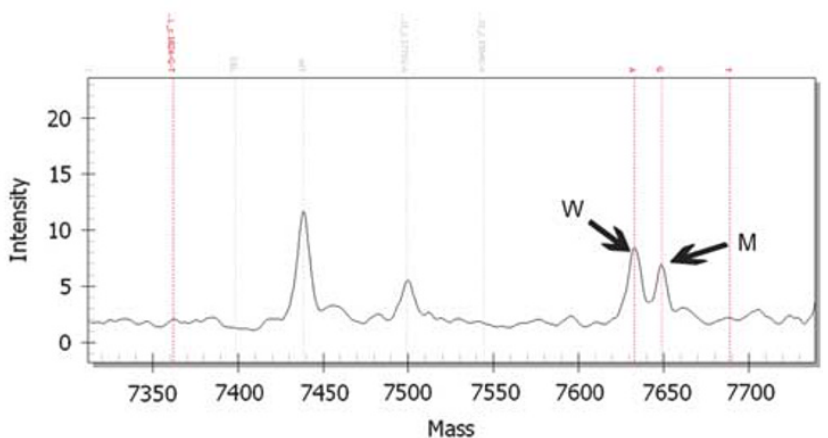

Figure 2 Encapsulated follicular variant of papillary thyroid carcinoma. (a) Low-power view showing tumor (T) surrounded by its capsule (cap) with no invasion. (b) Medium-power view revealing follicular growth with densely eosinophilic colloid characteristic of the follicular variant of papillary thyroid carcinoma. (c) High-power view of the nuclear features of papillary thyroid carcinoma: hypochromatic, irregular and overlapping nuclei with grooves (the latter indicated by an arrow). (d) Mass spectrometry traces for NRAS codon 61 mutation from the tumor. Note the mutant NRAS $182 \mathrm{G}$ (indicated by M) and wild-type (W) peaks.

to 12 and 13 of RET, which flank the rearrangement site in intron 11. Samples with 12-13 >10-11 expression were screened for specific RET recombination events using primers bracketing the fusion point of RET/PTC1, RET/PTC2 and RET/PTC3, respectively, as previously described. ${ }^{7,8}$ We screened for the PAX8/PPAR $\gamma$ fusion by reverse transcriptase-PCR, using primers for the different possible transcripts of $P A X 8 / P P A R \gamma$ as previously described. ${ }^{9}$ PCR products were resolved by electrophoresis in a $2 \%$ agarose gel and selected cases were sequenced. $R E T$ and $P A X 8 / P P A R \gamma$ rearrangements were analyzed for tumors that were wild type for $R A S$ and BRAF.

\section{Statistical Analysis}

Two-tailed Fisher's exact test was used to assess the relation between categorical variables. The $P$-value of $<0.05$ was considered as significant.

\section{Results}

\section{Clinical and Histopathological Features}

A total of 47 cases were included in the study (19 infiltrative and 28 encapsulated follicular variant). All captured cases of infiltrative follicular variant with adequate tissue were analyzed, whereas the encapsulated tumors were randomly selected. This randomization was performed in view of the large number of encapsulated follicular variant of papillary thyroid carcinomas in the database.

Table 1 lists the clinical and pathological features according to the invasive growth patterns of the follicular variant of papillary thyroid carcinoma (encapsulated vs infiltrative). There was no significant difference in age, gender, tumor size, mitotic rate or vascular invasion between patients with encapsulated tumors and those with infiltrative neoplasms. In contrast, patients with encapsulated carcinomas had a significantly lower rate of 
Table 1 Clinical and pathological characteristics according to the histological subvariants of the follicular variant of papillary thyroid carcinoma (encapsulated and nonencapsulated)

\begin{tabular}{ccc}
\hline \multirow{3}{*}{ Characteristic } & \multicolumn{2}{c}{ No. of patients (\%) } \\
\cline { 2 - 3 } & $\begin{array}{c}\text { Encapsulated } \\
\text { papillary thyroid } \\
\text { carcinoma, } \\
\text { follicular variant } \\
(\mathrm{n}=28 \text { patients })\end{array}$ & $\begin{array}{c}\text { Infiltrative } \\
\text { papillary thyroid } \\
\text { carcinoma, } \\
\text { follicular variant } \\
(\mathrm{n}=19 \text { patients })\end{array}$ \\
&
\end{tabular}

Age, years
Median

$\leq 45$

$>45$

47

$13(46)$

$15(54)$

Gender

Female

Male

$17(61)$

$11(39)$

2.9

Tumor size $(\mathrm{cm})$

Median

$\leq 4$

$>4$

$21(75)$

7 (25)

Vascular

invasion

Absent

Present

Capsular

invasion

Absent

Present

Extrathyroid

extension

Absent

Present

Margins

Absent

Present

Lymph node

metastases

Absent

Present

Distant

metastases

Absent

Present

Thyroid surgery

Lobectomy

Total

thyroidectomy

Radioactive

iodine therapy ${ }^{\mathrm{b}}$

No

Yes

$13(50)$

$13(50)$

7 (39)

$11(61)$
NA, not applicable.

${ }^{\mathrm{a}}$ Fisher's exact test, two-tailed values.

${ }^{\mathrm{b}}$ Radioactive iodine status was available in 26 encapsulated tumors and 18 infiltrative carcinomas. extrathyroid extension (4 vs 53\%, respectively, $P=0.0001$ ) and positive margins (4 vs $26 \%$, respectively, $P=0.03)$. The presence of any invasion (capsular or vascular) was observed in 6 of 29 $(21 \%)$ encapsulated follicular variant (2 tumors showed both capsular and vascular invasion, 2 tumors showed capsular invasion only and 2 tumors showed vascular invasion alone). Of 29 encapsulated carcinomas, $23(79 \%)$ were totally noninvasive. The lymph node metastatic rate was significantly higher in patients who had infiltrative tumors (10 of 19 patients; 53\%) compared with patients who had encapsulated neoplasms ( 2 of 28 patients; $7 \% ; P=0.001$ ). All 23 patients who had noninvasive, encapsulated follicular variant of papillary thyroid carcinoma lacked evidence of lymph node metastases, except one case with a cervical node micrometastasis. This micrometastatic deposit was controlateral to the noninvasive encapsulated papillary thyroid carcinoma, follicular variant, and ipsilateral to two papillary microcarcinomas that showed a histology similar to the micrometastatic focus. In all, 23 patients had lymph node tissue available for microscopic examination. In these 23 patients, the metastatic lymph node rate was significantly higher for patients who had infiltrative tumors (10 of 13 patients; $77 \%$ ) than for patients who had encapsulated carcinomas ( 2 of 10 patients; $20 \% ; P=0.01)$.

Of the 28 patients with encapsulated follicular variant, 10 patients underwent a lobectomy, and total thyroidectomy was performed in the remaining 18 patients. Of the 19 patients with infiltrative tumors, 6 underwent lobectomy and 13 underwent total thyroidectomy. Two patients with encapsulated follicular variant of papillary thyroid carcinoma underwent a formal neck dissection (1 selective and the other a central compartment dissection). Formal neck dissection was also performed in 9 infiltrative follicular variant of papillary thyroid carcinomas subdivided as follows: modified neck dissection $(n=4)$, extended neck dissection $(n=4)$ and selective neck dissection $(n=1)$. Radioactive iodine therapy information was available in 26 encapsulated tumors. Of these 26, radioactive iodine was administered in $13(50 \%)$. Of the 18 patients with infiltrative carcinomas and known radioactive iodine status, $11(61 \%)$ received radioactive iodine therapy.

Of the 23 encapsulated follicular variant of papillary thyroid carcinomas with adequate follow-up (median: 5.4 years, up to 12 years), only one patient developed recurrence. This 66-year-old male who presented with bone metastasis had an encapsulated tumor with extensive capsular and vascular invasion. He developed recurrences and died of disease approximately 3 years after initial diagnosis. None of the 19 encapsulated noninvasive follicular variant of papillary thyroid carcinomas with adequate follow-up (median: 5.4 years, up to 12 years) recurred. Of the 19 infiltrative carcinomas, 3 had 

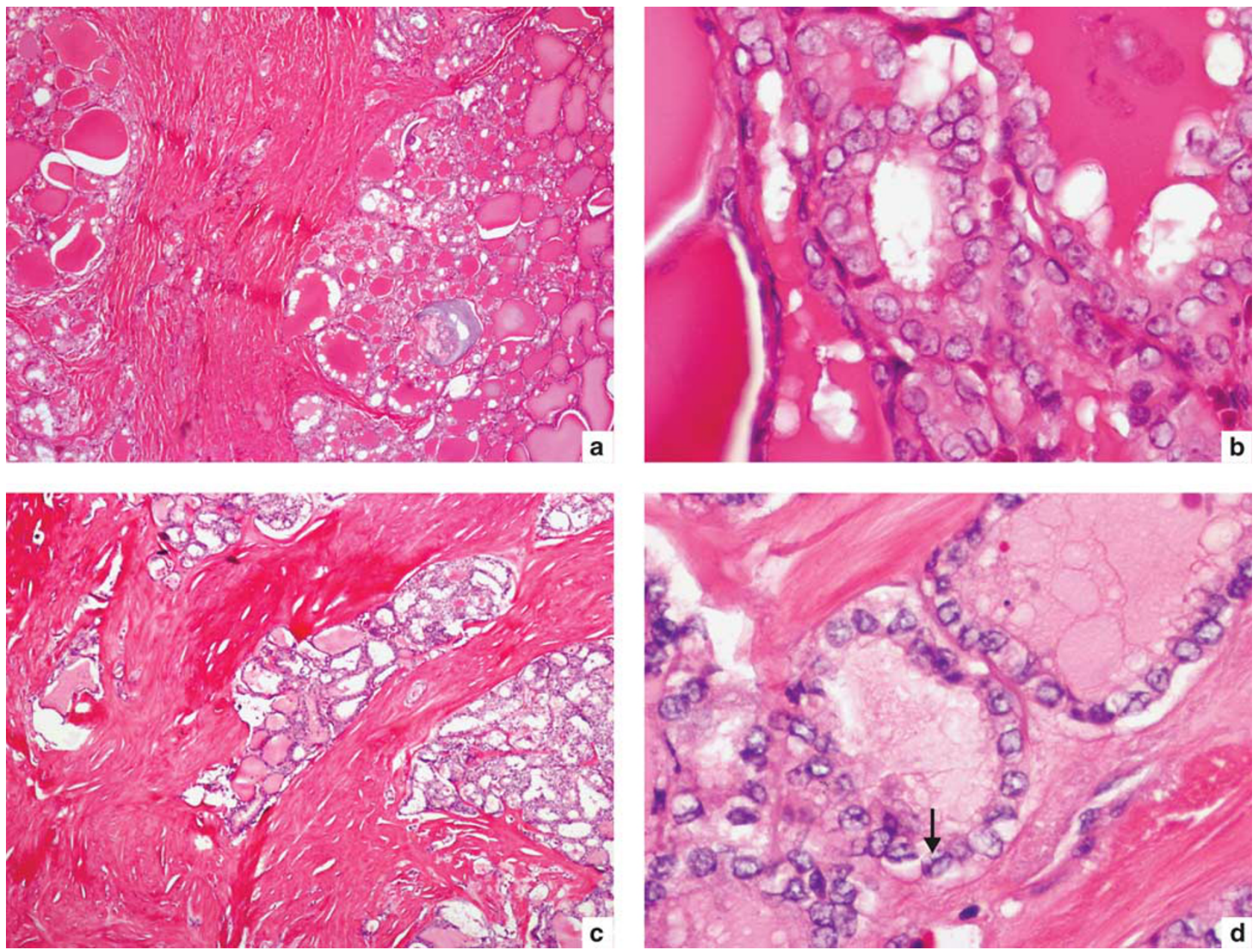

Figure 3 Infiltrative follicular variant of papillary thyroid carcinoma metastatic to lung at presentation. (a, b) A 35-year-old male with $B R A F_{-} T 1799 A$ mutation. Medium power of primary tumor (a) shows tumor growing in a follicular pattern with marked fibrosis. High power (b) reveals clear, irregular and overlapping nuclei. (c, d) A 53-year-old male with no mutations detected. Medium power of primary tumor (c) shows tongues of infiltrating tumor with marked fibrosis. High power of follicle lined by clear, irregular and overlapping nuclei with grooves (arrow).

distant metastases. All were present at presentation (one patient with bone and two with lung metastasis). Of the 16 infiltrative tumors with adequate follow-up (10 years, up to 19 years), one patient was alive with disease and the remainder free of disease. This patient with persistent disease had lung metastases at presentation (Figure 3). The other case with lung metastasis at presentation was lost for follow-up, whereas the patient with bone metastasis at presentation had his single clavicular metastasis resected and was free of disease 7 years after his surgery.

\section{Genotyping Analysis}

Overall, genotyping abnormalities were found in 11 (39\%) of 28 encapsulated follicular variant of papillary thyroid carcinomas and in $9(47 \%)$ of 19 infiltrative tumors. Table 2 lists the genotyping data according to the histological subtypes of the tumor.
There was a statistically higher $B R A F \quad 1799 T>A$ positivity rate in infiltrative carcinomas $(26 \%)$ than in encapsulated tumors $(0 \%)(P=0.007$; Figure 1$)$. In contrast, there was a trend toward higher $R A S$ positivity rate in encapsulated follicular variant of papillary thyroid carcinomas $(36 \%)$ when compared with infiltrative tumors $(10 \% ; P=0.09)$. Within encapsulated follicular variant of papillary thyroid carcinomas, the most common $R A S$ mutations were NRAS $182 A>G(Q 61 R)(n=5)$ followed by HRAS $182 A>G(Q 61 R) \quad(n=2)$, HRAS $181 C>A(Q 61 K)$ $(n=1)$, HRAS $37 G>T$ (G13C) $(n=1)$ and KRAS $37 G>T$ (G13C) $(n=1$; Figure 2). Infiltrative follicular variant of papillary thyroid carcinoma showed only NRAS $182 A>G$ mutations $(n=2)$. In tumors that were wild type for $R A S$ and $B R A F$, one encapsulated follicular variant of papillary thyroid carcinoma contained a $P A X 8 / P P A R \gamma$ rearrangement (Figure 4), whereas two infiltrative tumors harbored a $R E T / P T C$ recombination. One of the infiltrative carcinoma harbored a RET/PTC3 fusion, whereas 
Table 2 Molecular genotype according to the histological subvariants of follicular variant of papillary thyroid carcinoma (encapsulated and nonencapsulated)

No. of patients (\%)

Genotype

Encapsulated papillary

thyroid carcinoma, follicular variant $(\mathrm{n}=28$ patients)
Infiltrative papillary

thyroid carcinoma, follicular variant $(\mathrm{n}=19$ patients)
$\mathrm{P}$-value

$\begin{array}{cl}5 / 19(26) & 0.007 \\ 2 / 19(10) & 0.09 \\ 0 / 19 & 1 \\ 2 / 19(10) & 0.15 \\ 9 / 19(47) & 0.76\end{array}$

$B R A F$ mutation

RAS mutation

$P A X 8 / P P A R \gamma$ rearrangement

$R E T / P T C$ recombination

Total number of genotypic

aberrations

$0 / 28$
$10 / 28(36)$
$1 / 28(3.5)$
$0 / 28$
$11 / 28(39)$

$0 / 28$

$128(3.5)$

$11 / 28(39)$

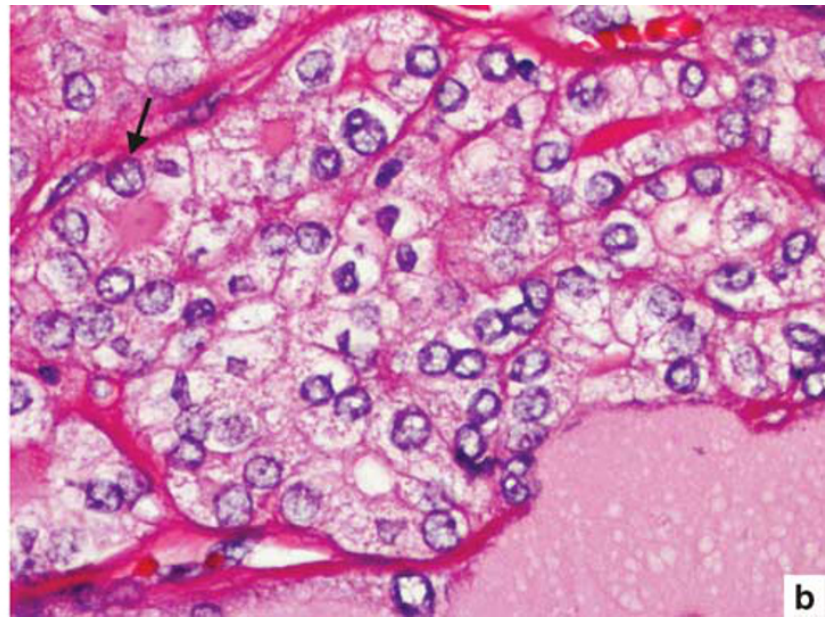

${ }^{\mathrm{a}}$ Fisher's exact test, two-tailed values.

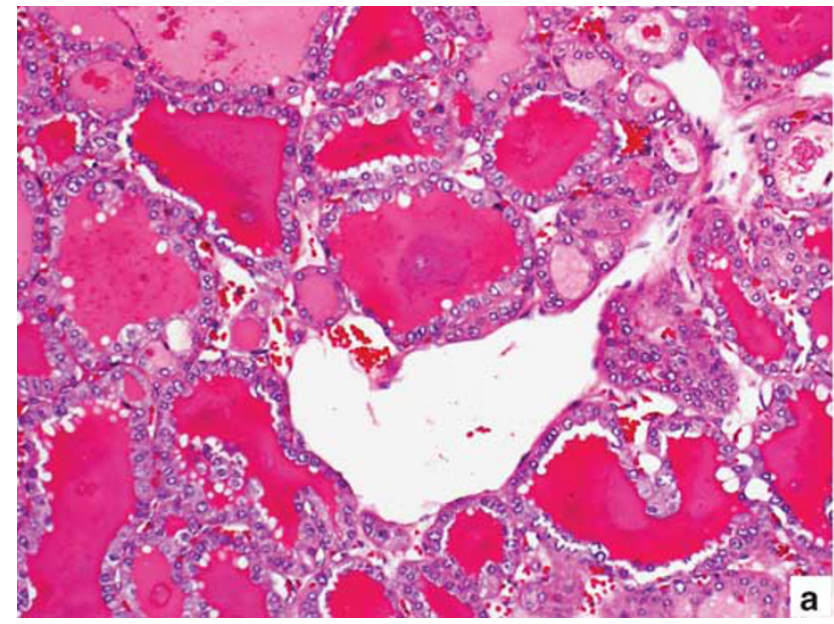

Figure 4 A 36-year-old male with encapsulated follicular variant of papillary thyroid carcinoma harboring a $P A X 8 / P P A R \gamma$ rearrangement. (a) Medium power showing follicles with densely eosinophilic colloid characteristic of the follicular variant of papillary thyroid carcinoma. (b) High power showing clear, irregular and overlapping nuclei with grooves (arrow).

the other showed a RET/PTC recombination event not detected by the specific reverse transcriptasePCR reactions for RET/PTC 1, 2 and 3. Within encapsulated follicular variant of papillary thyroid carcinomas, there was no correlation between the type of mutation and age, gender and vascular invasion. There was however a trend toward higher tumor size in $R A S$-mutated neoplasms (median: $4.25 \mathrm{~cm}$ for $R A S$-mutated tumors $v S 2.25 \mathrm{~cm}$ in non-RAS-mutated tumors, $P=0.06)$. Within infiltrative follicular variant of papillary thyroid carcinomas, there was no correlation between the type of mutation and age, gender, size, extrathyroid extension and nodal metastases. Within the whole patient population, $R A S$-mutated tumors had a significantly higher tumor size (median: $3.5 \mathrm{~cm}$ ) than non-RASmutated papillary thyroid carcinomas, follicular variant (median: $2 \mathrm{~cm}, P=0.018$ ). In addition, within the same group, there was a trend toward higher extrathyroid extension rate in $B R A F$-mutated papillary thyroid carcinomas, follicular variant $(3 / 5$, $60 \%)$ than in non-BRAF mutated tumors $(8 / 42$, $19 \% ; P=0.075)$. Within encapsulated follicular variant of papillary thyroid carcinomas, there was no correlation between genotype and outcome. The encapsulated follicular variant of papillary thyroid carcinoma with distant metastasis had a NRAS $182 A>G$ mutation. There was also no correlation between genotype and outcome in infiltrative follicular variant of papillary thyroid carcinomas. Of the two patients with infiltrative follicular variant of papillary thyroid carcinoma and lung metastasis at presentation, one had a BRAF $1799 T>A$ mutation, whereas the other case did not show any genetic aberration. The infiltrative case with bone metastasis at presentation showed an NRAS $182 A>G$ mutation.

\section{Discussion}

On the basis of the above data, encapsulated follicular variant of papillary thyroid carcinomas have a genotypic profile very close to the one observed in follicular adenoma/carcinoma. Indeed, $R A S$ mutations were found in $36 \%$ of encapsulated follicular variant of papillary thyroid carcinomas. This rate is very similar to the overall $R A S$ mutation 
frequency range observed in non-oncocytic follicular adenomas (24-53\%) and carcinomas (18-52\%). ${ }^{10-15}$ Furthermore, as described for follicular adenoma and carcinomas, NRAS codon 61 mutations were the most common $R A S$ abnormality observed in encapsulated follicular variant of papillary thyroid carcinomas. ${ }^{10}$ In contrast to $R A S$ mutations, BRAF 1799T $>A$ mutations and $R E T / P T C$ fusions were not found in encapsulated follicular variant of papillary thyroid carcinomas. This is again consistent with a follicular adenoma/carcinoma molecular profile, as these alterations are characteristically absent in follicular adenomas and carcinomas. ${ }^{16}$ Only one encapsulated follicular variant harbored a $P A X 8 / P P A R \gamma$ rearrangement in our series. This is closer to the findings of $\mathrm{Zhu}$ et al, ${ }^{17}$ who did not detect $P A X 8 / P P A R \gamma$ alteration in follicular variant of papillary thyroid carcinomas, but at odds with Castro et $a{ }^{16}{ }^{16}$ who detected this rearrangement in $37.5 \%$ of follicular variant of papillary thyroid carcinomas. These discrepant results could be due to differences in genotyping technologies or to different definitions and thresholds for the histological diagnosis of follicular variant of papillary thyroid carcinomas. It is interesting to note that the sole tumor with $P A X 8 / P P A R \gamma$ rearrangement in our study showed angioinvasion. Nikiforova et $a 1^{10}$ have shown that this fusion gene correlates with the presence of angioinvasion in follicular carcinomas and Castro et al ${ }^{16}$ showed the same relationship in the follicular variant of papillary thyroid carcinomas.

Although encapsulated follicular variant had a molecular profile very similar to the one observed in follicular adenoma/carcinoma, most of the genetic alterations found in infiltrative follicular variant of papillary thyroid carcinomas are in between follicular adenomas/carcinomas and classical papillary thyroid carcinomas, although closer to the latter. Indeed, $B R A F$ mutations were present in $26 \%$ of these tumors. This is definitely much higher than the $0 \%$ rate observed in follicular adenomas/ carcinomas but lower than classical papillary carcinomas $(57 \%) .^{5}$ The $R A S$ mutation frequency in infiltrative follicular variant of papillary thyroid carcinomas $(10 \%)$ was closer to classical papillary carcinomas $(0 \%)$ than to follicular carcinomas (average $40 \%)^{18}$ The absence of $P A X 8 / P P A R \gamma$ rearrangement in infiltrative follicular variant of papillary thyroid carcinoma is much more consistent with a classical papillary thyroid carcinoma genotype rather than a follicular adenoma/carcinoma molecular profile. Indeed, that alteration is virtually absent in papillary carcinomas $(0-1 \%)^{10,19-21}$ but present in a significant proportion of follicular adenomas/carcinomas. ${ }^{10}$ RET/PTC fusions were detected in $10 \%$ of infiltrative follicular variant of papillary thyroid carcinomas. This rate is in between the $0 \% R E T / P T C$ frequency observed in follicular adenoma/carcinomas and the 26\% RET/ PTC fusion rate reported in classical papillary thyroid carcinomas. ${ }^{5}$
As shown in our previous study on the subject, patients who had infiltrative follicular variant of papillary thyroid carcinoma in this study had a significantly greater frequency $(P<0.05)$ of extrathyroid extension, and positive margins than patients who had encapsulated follicular variant of papillary thyroid carcinoma. This superior potential of infiltrative follicular variant of papillary thyroid carcinoma in invading the extrathyroid stroma was reflected by its higher rate of regional lymph node metastases. Indeed, patients with infiltrative follicular variant of papillary thyroid carcinoma had a metastatic lymph node rate of $53 \%$ compared with $7 \%$ for patients with encapsulated follicular variant of papillary thyroid carcinoma $(P=0.001)$. This strong and significant correlation between lymph node metastases and infiltrative follicular variant of papillary thyroid carcinoma was maintained when only specimens (23 tumors) that contained lymph node tissue were analyzed. This difference in lymph node disease could not be explained by differences in tumor size or age at presentation, because the latter two variables were similar in both the encapsulated and infiltrative group. In this study, the metastatic lymph node pattern of encapsulated follicular variant of papillary thyroid carcinomas $(7 \%)$ was much closer to that reported in follicular carcinomas (on the order of 5-10\%), whereas infiltrative follicular variant of papillary thyroid carcinomas had a metastatic lymph node pattern within the range reported for classic papillary carcinomas (on the order of $45-65 \%)^{17,22}$

With regard to prognosis, patients who had invasive tumors, whether encapsulated or not, had a rare but real potential for adverse outcome. One patient who had an encapsulated follicular variant of papillary thyroid carcinoma with extensive capsular and vascular invasion harbored distant metastasis at presentation, developed recurrences and died of disease approximately 3 years after initial diagnosis. Interestingly, this patient did not develop any regional nodal disease, consistent with the behavior of follicular carcinoma rather than papillary carcinoma. ${ }^{23}$ In contrast, none of the 19 patients with adequate follow-up who had noninvasive, encapsulated follicular variant of papillary thyroid carcinoma developed recurrences, or died of disease. These data are in congruence with our previous reports stating the indolent nature of encapsulated non-invasive follicular variant of papillary thyroid carcinoma. ${ }^{2,24}$ Indeed, encapsulated follicular variant of papillary thyroid carcinoma appears to behave similar to follicular adenoma/ carcinoma, its metastatic potential governed by the presence or absence of capsular or vascular invasion. In addition to the correlation between the overall molecular genotype of follicular variant of papillary thyroid carcinoma subtype and their respective behavior, we found a relationship between some individual mutations and clinicopathological features. In accordance with previous articles on follicular variant of papillary thyroid carcinomas, 
the presence of RAS significantly correlated with large tumor size within our whole patient population. $(P<0.05) .{ }^{16}$ In addition, within the same group, there was a trend toward higher extrathyroid extension rate in $B R A F$-mutated follicular variant of papillary thyroid carcinomas $(60 \%)$ than in non$B R A F$-mutated tumors $(19 \% ; P=0.075)$. The association between BRAF and higher extrathyroid extension rate in papillary carcinoma has been shown by us and others. ${ }^{5,25}$

In conclusion, this study shows that encapsulated and infiltrative follicular variant of papillary thyroid carcinomas have different genotypic profiles. Encapsulated follicular variant of papillary thyroid carcinoma has a $B R A F$ and $R A S$ mutation pattern very similar to follicular adenoma/carcinoma, whereas infiltrative follicular variant of papillary thyroid carcinoma has a BRAF and RAS genotype in between follicular adenoma/carcinoma and classical papillary carcinoma, although closer to the latter (Figure 5). The molecular profile of each follicular variant of papillary thyroid carcinoma subtype mirrors their histopathological features and metastatic behavior. This strongly argues for follicular variant of papillary thyroid carcinoma being two diseases: (1) encapsulated follicular variant of papillary thyroid carcinoma with a genotypic, invasive and behavioral profile very close to follicular adenoma/carcinoma and (2) infiltrative follicular variant of papillary thyroid carcinoma with invasive and behavioral features very close to classical papillary carcinoma and a molecular profile in between follicular adenoma/carcinoma and classical papillary carcinoma, although closer to the latter.

These findings may have important clinical implications. As encapsulated follicular variant of papillary thyroid carcinoma has a genotypic profile as well as invasive and behavioral features very similar to follicular adenoma/carcinoma, one may reconsider reclassifying it as an entity close to the follicular adenoma/carcinoma group. The same criteria that are used to decide whether follicular tumors are

\begin{tabular}{ll}
$\begin{array}{l}\text { Follicular adenoma/ } \\
\text { carcinoma (low rate of } \\
\text { nodal metastasis) }\end{array}$ & \multicolumn{1}{c}{\begin{tabular}{l} 
Classical papillary \\
carcinoma (high rate \\
of nodal metastasis) \\
\hline$R A S+++$
\end{tabular}} \\
\hline $\begin{array}{l}\text { Encapsulated follicular } \\
\text { variant papillary carcinoma } \\
\text { (low rate of nodal metastasis) }\end{array}$ & $\begin{array}{l}\text { Infiltrative } \\
\text { follicular variant papillary } \\
\text { carcinoma (high rate of nodal } \\
\text { metastasis) }\end{array}$
\end{tabular}

Figure $5 B R A F$ and $R A S$ mutational patterns of follicular variant of papillary thyroid carcinoma according to its encapsulated and infiltrative subtypes. The mutational pattern of encapsulated follicular variant of papillary thyroid carcinoma is similar to follicular adenoma/carcinoma, whereas infiltrative follicular variant of papillary thyroid carcinoma has a $B R A F$ and $R A S$ genotype in between follicular adenoma/carcinoma and classical papillary carcinoma, although closer to the latter. The mutational patterns of encapsulated and infiltrative follicular variant of papillary thyroid carcinomas parallel their lymph node metastatic rate. biologically benign or malignant (ie, capsular and vascular invasion) would be applied to the evaluation of encapsulated follicular variant of papillary thyroid carcinoma. In practical terms, a lack of capsular and vascular invasion should denote an extremely indolent clinical behavior in encapsulated follicular variant of papillary thyroid carcinoma. If this reclassification is achieved, it will have a major effect on the diagnosis and management of patients with follicular variant of papillary thyroid carcinoma. In noninvasive, encapsulated follicular variant of papillary thyroid carcinoma, pathologists will be spared the frustrating and very subjective exercise of deciding whether a tumor has the nuclear features of papillary carcinoma. More importantly, countless numbers of patients with noninvasive, encapsulated follicular variant of papillary thyroid carcinoma will be treated conservatively akin minimally invasive follicular carcinoma. These individuals will be spared unnecessary and aggressive surgical and radioactive iodine therapy with their attached morbidity and financial costs.

\section{Disclosure/conflict of interest}

The authors declare no conflict of interest.

\section{References}

1 Rosai J, Carcangiu ML, Delellis RA. Tumors of the thyroid gland In: Rosai J, Sobin LH (eds). Atlas of Tumor Pathology, Vol. 5. Armed Forces Institute of Pathology: New York, 1992, pp 161-182.

2 Liu J, Singh B, Tallini G, et al. Follicular variant of papillary thyroid carcinoma: a clinicopathologic study of a problematic entity. Cancer 2006;107:1255-1264.

3 Lloyd RV, Erickson LA, Casey MB, et al. Observer variation in the diagnosis of follicular variant of papillary thyroid carcinoma. Am J Surg Pathol 2004;28:1336-1340.

4 Elsheikh TM, Asa SL, Chan JK, et al. Interobserver and intraobserver variation among experts in the diagnosis of thyroid follicular lesions with borderline nuclear features of papillary carcinoma. Am J Clin Pathol 2008;130:736-744.

5 Adeniran AJ, Zhu Z, Gandhi M, et al. Correlation between genetic alterations and microscopic features, clinical manifestations, and prognostic characteristics of thyroid papillary carcinomas. Am J Surg Pathol 2006;30:216-222.

6 Lang W, Choritz H, Hundeshagen H. Risk factors in follicular thyroid carcinomas. A retrospective followup study covering a 14-year period with emphasis on morphological findings. Am J Surg Pathol 1986;10:246-255.

7 Ricarte-Filho JCM, Ryder M, Chitale DA, et al. Mutational profile of advanced primary and metastatic radioactive iodine-refractory thyroid cancers reveals distinct pathogenetic roles for BRAF, PIK3CA and AKT1. Cancer Res 2009;69:4885-4893.

8 Imkamp F, von Wasielewski R, Musholt TJ, et al. Rearrangement analysis in archival thyroid tissues: 
punching microdissection and artificial RET/PTC 1-12 transcripts. J Surg Res 2007;143:350-363.

9 Nikiforova MN, Biddinger PW, Caudill CM, et al. PAX8-PPARgamma rearrangement in thyroid tumors: RT-PCR and immunohistochemical analyses. Am J Surg Pathol 2002;26:1016-1023.

10 Nikiforova MN, Lynch RA, Biddinger PW, et al. RAS point mutations and PAX8-PPARgamma rearrangement in thyroid tumors: evidence for distinct molecular pathways in thyroid follicular carcinoma. J Clin Endocrinol Metab 2003;88:2318-2326.

11 Lemoine NR, Mayall ES, Wyllie FS, et al. High frequency of ras oncogene activation in all stages of human thyroid tumorigenesis. Oncogene 1989;4:159-164.

12 Suarez HG, du Villard JA, Severino M, et al. Presence of mutations in all three ras genes in human thyroid tumors. Oncogene 1990;5:565-570.

13 Esapa CT, Johnson SJ, Kendall-Taylor P, et al. Prevalence of Ras mutations in thyroid neoplasia. Clin Endocrinol (Oxf) 1999;50:529-535.

14 Motoi N, Sakamoto A, Yamochi T, et al. Role of ras mutation in the progression of thyroid carcinoma of follicular epithelial origin. Pathol Res Pract 2000;196:1-7.

15 Namba H, Rubin SA, Fagin JA. Point mutations of ras oncogenes are an early event in thyroid tumorigenesis. Mol Endocrinol 1990;4:1474-1479.

16 Castro P, Rebocho AP, Soares RJ, et al. PAX8-PPARgamma rearrangement is frequently detected in the follicular variant of papillary thyroid carcinoma. J Clin Endocrinol Metab 2006;91:213-220.

17 Zhu Z, Gandhi M, Nikiforova MN, et al. Molecular profile and clinical-pathologic features of the follicular variant of papillary thyroid carcinoma. An unusually high prevalence of ras mutations. Am J Clin Pathol 2003;120:71-77.

18 Nikiforov YE. Thyroid carcinoma: molecular pathways and therapeutic targets. Mod Pathol 2008;21(Suppl 2): S37-S43.

19 Nikiforova MN, Biddinger PW, Caudill CM, et al. PAX8-PPARgamma rearrangement in thyroid tumors: RT-PCR and immunohistochemical analyses. Am J Surg Pathol 2002;26:1016-1023.

20 Marques AR, Espadinha C, Catarino AL, et al. Expression of PAX8-PPARgamma 1 rearrangements in both follicular thyroid carcinomas and adenomas. J Clin Endocrinol Metab 2002;87:3947-3952.

21 French CA, Alexander EK, Cibas ES, et al. Genetic and biologic subgroups of early stage follicular thyroid cancer. Am J Pathol 2003;162:1053-1060.

22 Passler C, Prager G, Scheuba C, et al. Follicular variant of papillary thyroid carcinoma: a long-term follow-up. Arch Surg 2003;138:1362-1366.

23 Baloch ZW, LiVolsi VA. Encapsulated follicular variant of papillary thyroid carcinoma with bone metastases. Mod Pathol 2000;13:861-865.

24 Rivera M, Tuttle RM, Patel S, et al. Encapsulated papillary thyroid carcinoma: a clinico-pathologic study of 106 cases with emphasis on its morphologic subtypes (histologic growth pattern). Thyroid 2009;19: 119-127.

25 Rivera M, Ricarte-Filho J, Knauf J, et al. Molecular and morphologic characterization of thyroid carcinomas according to extra-thyroid extension status. Mod Pathol 2010;23(Suppl 1S):132A (Abst 584). 